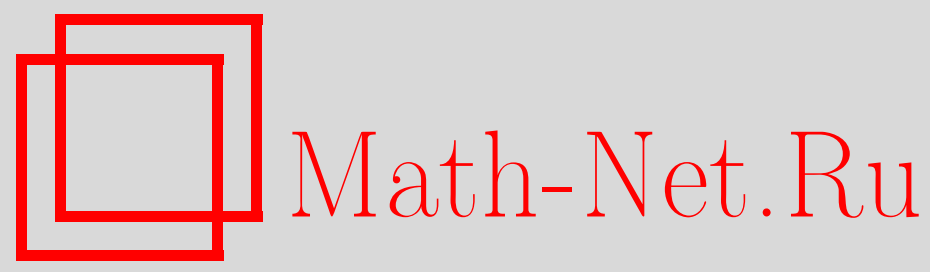

В. И. Парусников, Многогранники Клейна для трех экстремальных кубических форм, Матем. заметки, 2005, том 77, выпуск 4, 566-583

DOI: https://doi.org/10.4213/mzm2518

Использование Общероссийского математического портала Math-Net.Ru подразумевает, что вы прочитали и согласны с пользовательским соглашением http://www . mathnet.ru/rus/agreement

Параметры загрузки:

IP : 52.6 .47 .48

26 апреля 2023 г., 18:05:39

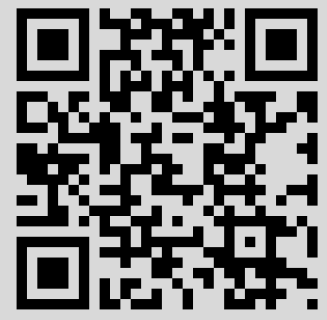




\title{
МНОГОГРАННИКИ КЛЕЙНА \\ ДЛЯ ТРЕХ ЭКСТРЕМАЛЬНЫХ КУБИЧЕСКИХ ФОРМ
}

\section{В. И. Парусников}

\begin{abstract}
Давенпортом и Свиннертоном-Дайером были найдены первые 19 экстремальных тернарных кубических форм $g_{i}$, имеюших тот же смысл, что и известные формы Маркова в бинарном квадратичном случае. Многогранники Клейна для форм $g_{1}-g_{4}$ были недавно вычислены Брюно и Парусниковым. Они же для кратных корневых векторов вычислили "подходящие дроби", полученные по разным матричным обобщениям алгоритма цепных дробей, и изучили их расположение относительно многогранников Клейна. В настоящей статье вычислены многогранники Клейна форм $g_{5}-g_{7}$ и сопряженной формы $g_{7}^{*}$. Найдены их периоды и фундаментальные области. Вычислены разложения кратных корневых векторов этих форм по матричным алгоритмам Эйлера, Якоби, Пуанкаре, Бруна, Парусникова, Брюно. По расположению "подходяших дробей" относительно многогранников Клейна оценивалось качество алгоритма. С этой точки зрения алгоритмы Эйлера и Пуанкаре оказались наихудштми, а алгоритм Брюно наилучшим. Но ни один из этих алгоритмов не обобщает все свойства цепной дроби.
\end{abstract}

Библиография: 21 название.

1. Введение. Наилучшие рациональные приближения $p / q$ к числу $\alpha$ суть подходящие дроби $p_{k} / q_{k}$ цепной дроби числа $\alpha[1]$. Клейн [2]-[4] предложил такую интерпретацию цепных дробей (см. также [5, гл. III, п. 19]). Пусть на плоскости координат $p, q$ проведены прямые $\mathscr{L}_{1}=\{p, q: p=\alpha q\}$ и $\mathscr{L}_{2}=\{p, q: q=0\}$. Они образуют смежные углы $\mathscr{O}_{1}=\{p, q: p \geqslant \alpha q, q \geqslant 0\}$ и $\mathscr{O}_{2}=\{p, q: p \leqslant \alpha q, q \geqslant 0\}$. Обозначим $K_{j}$ выпуклую оболочку целых точек $(p, q) \in \mathbb{Z}^{2}$ без нуля, попавших в $\mathscr{O}_{j}$. Границы $\partial K_{j}$ множеств $K_{j}$ суть вьпуклые ломаные линии, вершинам $(p, q)$ которых отвечают подходящие дроби $p / q$ цепной дроби числа $\alpha$.

Через ${ }^{T} M$ обозначим транспонирование матрицы $M$, a $(M)_{k, l}$ - элемент eе $l$-го столбца $k$-й строки. Линейные формы будем записьвать как произведение $L X$ матриц: вектор-строки $L=\left(l_{1}, \ldots, l_{n}\right) \in \mathbb{R}_{*}^{n}$ на вектор-столбец $X={ }^{T}\left(x_{1}, \ldots, x_{n}\right) \in \mathbb{R}^{n}$.

В [3], [4] Клейн предложил также и трехмерную конструкцию для изучения наилучших приближений. Три пересекающиеся в нуле плоскости $\mathscr{L}_{j}=\left\{X: L_{j} X=0\right\}$ делят $\mathbb{R}^{3}$ на 8 октантов $\mathscr{O}_{\Sigma}=\left\{X: \sigma_{j} L_{j} X \geqslant 0, j=1,2,3\right\}\left(\Sigma=\left(\sigma_{1}, \sigma_{2}, \sigma_{3}\right)\right.$ - набор знаков $\left.\sigma_{j}= \pm 1\right)$. Замыкание $K_{\Sigma}$ выпуклой оболочки целых точек $X \in \mathscr{O}_{\Sigma}, X \neq 0$, ограничено

Работа выполнена при финансовой поддержке Российского фонда фундаментальных исследований, гранты № 03-01-00027, № 02-01-01067, № 05-01-00050, и программы ОММ РАН. 
выпуклой двумерной многогранной поверхностью $\partial K_{\Sigma}$, состоящей из вершин, ребер и граней. Ее вершины дают в некотором смысле наилучшие рациональные приближения линейных форм $L_{j} X$.

Для кубической однородной разложимой формы $g(X)=L_{1} X \cdot L_{2} X \cdot L_{3} X$ обозначим через $\Delta$ определитель матрицы, составленной из $L_{1}, L_{2}, L_{3}$. Давенпорт [6]-[8] доказал, что следующая величина:

$$
\mu(g) \stackrel{\text { def }}{=} \frac{1}{|\Delta|} \min _{X \in \mathbb{Z}^{3} \backslash\{0\}}|g(X)|
$$

не превосходит $1 / 7$ и $\mu(g)=1 / 7$ только для формы $g_{1}$, у которой $L_{j}=\left(1, \lambda_{j}, \lambda_{j}^{2}\right)$, где $\lambda_{j}$ суть корни уравнения $\lambda^{3}+\lambda^{2}-2 \lambda-1=0$, и для эквивалентных форм. Если их исключить из рассмотрения, то $\mu(g) \leqslant 1 / 9$ и $\mu(g)=1 / 9$ лишь для формы $g_{2}$ с $L_{j}=\left(1, \lambda_{j}, \lambda_{j}^{2}\right)$, где теперь $\lambda_{j}$ - корни уравнения $\lambda^{3}-3 \lambda-1=0$, и для эквивалентных форм. Свиннертон-Дайер [9] отыскал первые 19 форм $g_{i}$ с наибольшими значениями $\mu$.

В статьях [10]-[13] для форм $g_{1}-g_{4}$ были вычислены многогранники Клейна и разложения векторов $L_{3}$ по матричным алгоритмам Эйлера, Якоби, Пуанкаре, Бруна, Брюно, Парусникова. Изучалось расположение их "подходящих дробей" относительно многогранников Клейна. В препринтах [14]-[16] это же было сделано для форм $g_{5}-g_{7}$. Методом Эрмита форму $g_{1}$ изучал Шарв [17], а ее многогранники Клейна вычисляла Коркина [18].

В настоящей статье вычисляются многогранники Клейна форм $g_{5}-g_{7}$ и разложения их кратных векторов $L_{3}$ по матричньм алгоритмам. По расположению "подходящих дробей” относительно многогранников Клейна оценивалось качество алгоритма. С этой точки зрения алгоритмы Эйлера и Пуанкаре оказались наихудшими, а алгоритм Брюно наилучшим. Но ни один из этих алгоритмов не обобщает все свойства цепной дроби. Подробные таблицы, иллюстрирующие предлагаемьй материал приведены в препринтах [14]-[16].

2. Многогранники Клейна. О выпуклых многогранниках и конусах см. [19], [20]. Напомним определения, данные в [12]. Рассмотрим два $n$-мерных вешественных пространства $\mathbb{R}^{n}$ и $\mathbb{R}_{*}^{n}$ с координатами $x_{1}, \ldots, x_{n}$ и $u_{1}, \ldots, u_{n}$ соответственно. Произведение $U X=x_{1} u_{1}+\cdots+u_{n} x_{n}$ матрищ $U=\left(u_{1}, \ldots, u_{n}\right)$ и $X={ }^{T}\left(x_{1}, \ldots, x_{n}\right)$ задает операцию спаривания пространств. Базисы $L_{1}, \ldots, L_{n} \in \mathbb{R}_{*}^{n}$ и $M_{1}, \ldots, M_{n} \in \mathbb{R}^{n}$ назовем сопряженными, если $L_{k} M_{j}=\delta_{k, j}, k, j=1, \ldots, n$, где $\delta_{k, j}$ - символ Кронекера. Отвечающие векторам $L_{j}$ гиперплоскости $\mathscr{L}_{j}=\left\{X: L_{j} X=0\right\}$ делят пространство $\mathbb{R}^{n}$ на вьпуклые конусы. Каждому из $2^{n}$ наборов знаков $\Sigma=\left(\sigma_{1}, \ldots, \sigma_{n}\right)$, $\sigma_{j}= \pm 1$, соответствует конус $\mathscr{O}_{\Sigma}=\left\{X: \sigma_{j} L_{j} X \geqslant 0, j=1, \ldots, n\right\}$ (вместо +1 и -1 мы будем писать + и - ). Аналогично в $\mathbb{R}_{*}^{n}$ определяются двойственные конусы $\mathscr{O}_{\Sigma}^{*}=\left\{U: \sigma_{j} U M_{j} \geqslant 0, j=1, \ldots, n\right\}$.

Многогранником Клейна $K_{\Sigma}$ назовем вьпуклую оболочку множества ненулевых целых точек $X \in \mathbb{Z}^{n} \backslash\{0\}$ конуса $\mathscr{O}_{\Sigma}$. Аналогичное подмножество $K_{\Sigma}^{*}$ конуса $\mathscr{O}_{\Sigma}^{*}$ назовем многогранником Клейна, двойственным $K_{\Sigma}^{*}$. Ограничивающие многогранники $(n-1)$-мерные поверхности $\partial K_{\Sigma}$ и $\partial K_{\Sigma}^{*}$ состоят из граней $\Gamma_{s}^{(d)}$ разных размерностей $d$, $0 \leqslant d<n-1$ : вершин $(d=0)$, ребер $(d=1), \ldots$, гиперграней $(d=n-1)$. В [12] приведен алгоритм, которьм во многих случаях удается вычислить все целые точки поверхностей многогранников Клейна. 
Пусть целые точки $X_{1}, \ldots, X_{n}$ лежат в гиперплоскости $\mathscr{L}$, содержащей грань $\Gamma_{s}^{(n-1)}$, и пусть векторы $X_{j}-X_{1}, i=2, \ldots, n$, образуют целочисленный базис гиперплоскости $\mathscr{L}$, т.е. у составленной из этих векторов $(n-1) \times n$-матрицы наибольший общий делитель всех миноров порядка $n-1$ равен 1 . Величину $\rho\left(\Gamma_{s}^{(n-1)}\right) \stackrel{\operatorname{def}}{=}\left|\operatorname{det}\left(X_{1} X_{2} \cdots X_{n}\right)\right|$ назовем расстоянием гиперграни $\Gamma_{s}^{(n-1)}$. Относительным минимумом назовем точку $X_{0} \in \mathbb{Z}^{n} \cap \mathscr{O}_{\Sigma}$, для которой в октанте $\mathscr{O}_{\Sigma}$ нет ненулевых целых точек $X$ со свойством

$$
\left|L_{j} X\right|<\left|L_{j} X_{0}\right|, \quad j=1, \ldots, n
$$

3. Формы $g_{5}-g_{7}$. Зафиксируем индекс $i$ экстремальной формы $g_{i}$ СвиннертонаДайера [9] (этот индекс иногда будет опускаться). Форма $g_{i}$ задается трехмерной решеткой. Образующие решетки суть векторы $L_{i, j}=\left(1, \alpha_{i, j}, \beta_{i, j}\right), j=1,2,3$, с компонентами - алгебраически сопряженными числами из сопряженных вполне вещественных кубических полей $\mathbb{Q}\left(\lambda_{i, j}\right):$ числа $\lambda_{i, j} \in \mathbb{R}$ - корни неприводимого уравнения

$$
f_{i}=f_{i}(\lambda) \stackrel{\text { def }}{=} \lambda^{3}+f_{i, 1} \lambda^{2}+f_{i, 2} \lambda+f_{i, 3}=0, \quad f_{i, j} \in \mathbb{Z} .
$$

Для начальньх экстремальных форм алгебраические поля $\mathbb{Q}\left(\lambda_{i, j}\right)$ нормальнь (совпадают со своими сопряженными $\left.\mathbb{Q}\left(\lambda_{i, 1}\right)=\mathbb{Q}\left(\lambda_{i, 2}\right)=\mathbb{Q}\left(\lambda_{i, 3}\right)=K_{i}\right)$. Многогранники Клейна таких форм устроены сходно; проиллюстрируем их особенности напримере трех форм $g_{5}-g_{7}$.

Занумеруем корни полиномов (3) в порядке возрастания:

$$
\begin{array}{ll}
f_{5}=\lambda^{3}+\lambda^{2}-4 \lambda+1=0, & \left(\lambda_{5,1}, \lambda_{5,2}, \lambda_{5,3}\right) \approx(-2.6511, .2739,1.3772) \\
f_{6}=\lambda^{3}+4 \lambda^{2}-25 \lambda-1=0, & \left(\lambda_{6,1}, \lambda_{6,2}, \lambda_{6,3}\right) \approx(-7.3726,-.0397,3.4123), \\
f_{7}=\lambda^{3}-2 \lambda^{2}-\lambda+1=0, & \left(\lambda_{7,1}, \lambda_{7,2}, \lambda_{7,3}\right) \approx(-.8019, .5550,2.2470) .
\end{array}
$$

Векторы, задаюшие экстремальные формы, равны

$$
L_{i, j}= \begin{cases}\left(1, \lambda_{5, j},-2+2 \lambda_{5, j}+\lambda_{5, j}^{2}\right), & i=5, \\ \left(1, \frac{-5+3 \lambda_{6, j}+2 \lambda_{6, j}^{2}}{21}, \frac{-13+12 \lambda_{6, j}+\lambda_{6, j}^{2}}{21}\right), & i=6, \quad j=1,2,3 . \\ \left(1, \lambda_{7, j}^{2},-2 \lambda_{7, j}+\lambda_{7, j}^{2}\right), & i=7,\end{cases}
$$

Составим из вектор-строк $L_{i, j}, j=1,2,3$, матрищы $L_{i}$. Базисы, сопряженные к базисам $L_{i, j}, j=1,2,3$, задаются вектор-столбцами - столбцами $\widetilde{L}_{i, j}, j=1,2,3$, матриц $L_{i}^{-1}$ :

$$
\begin{gathered}
L_{i, j}=e_{j} L_{i}, \quad \widetilde{L}_{i, j}=L_{i}^{-1 T} e_{j}, \quad j=1,2,3, \\
e_{1} \stackrel{\text { def }}{=}(1,0,0), \quad e_{2} \stackrel{\text { def }}{=}(0,1,0), \quad e_{3} \stackrel{\text { def }}{=}(0,0,1) .
\end{gathered}
$$

Определим $\lambda_{i, j}$ для всех $j \in \mathbb{Z}$, положив $\lambda_{i, j}=\lambda_{i, k}$ для $j$, сравнимых с $k \in\{1,2,3\}$ по модулю 3 . Введем $s_{i, j}$ и ряд матриц: матрицу перестановок $J$, присоединенную матpuиy $A_{i, 0}$, задающие модуль и решетку матрищы $B_{i}$ и $A_{i}$, диагональную матрищу $\Lambda_{i}$, 
матрицу Вандермонда $W_{i}$, ганкелевы матрицы $S_{i, j}$ и симметричную матрищу $V_{i}$ :

$$
\begin{gathered}
J=\left(\begin{array}{lll}
0 & 1 & 0 \\
0 & 0 & 1 \\
1 & 0 & 0
\end{array}\right), \quad A_{i, 0}=\left(\begin{array}{ccc}
0 & 0 & -f_{i, 3} \\
1 & 0 & -f_{i, 2} \\
0 & 1 & -f_{i, 1}
\end{array}\right), \\
B_{5}=\left(\begin{array}{ccc}
1 & 0 & -2 \\
0 & 1 & 2 \\
0 & 0 & 1
\end{array}\right), \quad B_{6}=\frac{1}{21}\left(\begin{array}{ccc}
21 & -5 & -13 \\
0 & 3 & 12 \\
0 & 2 & 1
\end{array}\right), \quad B_{7}=\left(\begin{array}{ccc}
1 & 0 & 0 \\
0 & 0 & -2 \\
0 & 1 & 1
\end{array}\right), \quad\left(V_{i}\right)_{k, l}=\lambda_{i, k+l}, \\
A_{i}=B_{i}^{-1} A_{i, 0} B_{i}, \quad \Lambda_{i}=\operatorname{diag}\left\{\lambda_{i, k}\right\}_{k=1}^{3}, \quad\left(W_{i}\right)_{k, l}=\lambda_{i, k}^{l}, \quad j \in \mathbb{Z}, \quad k, l=1,2,3 .
\end{gathered}
$$

Отметим следуюшие свойства этих матриц: $L_{i}=W_{i} B_{i}, L_{i} A_{i}=\Lambda_{i} L_{i}, A_{i} L_{i}^{-1}=L_{i}^{-1} \Lambda_{i}$, $W_{i} A_{i, 0}=\Lambda_{i} W_{i}, S_{i, j}={ }^{T} W_{i} \Lambda_{i}^{k} W_{i}=S_{i, j-1} A_{i, 0}$, откуда следует рекуррентная формула Ньютона

$$
s_{i, j}=-\sum_{k=1}^{3} f_{i, k} s_{i, j-k}, \quad j \geqslant 3, \quad s_{i, 0}=3, \quad s_{i, 1}=-f_{i, 1}, \quad s_{i, 2}=f_{i, 1}^{2}-2 f_{i, 2} .
$$

Компоненты столбца $\widetilde{L}_{i, j}$ матрицы $L_{i}^{-1}=B_{i}^{-1} S_{i, 0}^{-1} W_{i}$ лежат в том же поле $\mathbb{Q}\left(\lambda_{i, j}\right)$, где и компоненты строки $L_{i, j}$. В наших случаях

$$
\begin{aligned}
& \widetilde{L}_{5, j}={ }^{T}\left(7-\lambda_{5, j}-\lambda_{5, j}^{2}, 3-\lambda_{5, j}^{2},-5+3 \lambda_{5, j}+2 \lambda_{5, j}^{2}\right) / 13 \\
& \widetilde{L}_{6, j}={ }^{T}\left(217-21 \lambda_{6, j}-7 \lambda_{6, j}^{2},-86+18 \lambda_{6, j}+5 \lambda_{6, j}^{2},-52+27 \lambda_{6, j}+4 \lambda_{6, j}^{2}\right) / 273 \\
& \widetilde{L}_{7, j}={ }^{T}\left(10+4 \lambda_{7, j}-4 \lambda_{7, j}^{2},-2+\lambda_{7, j}^{2},-2-6 \lambda_{7, j}+3 \lambda_{7, j}^{2}\right) / 14
\end{aligned}
$$

Одни корни уравнения (3) представляются как полиномы $\lambda_{j}=\phi_{k}\left(\lambda_{j-k}\right)$ от других корней, или $V=W \Phi$, где $\Phi=W^{-1} V=S_{0}^{-1}{ }^{T} W V$ - матрица коэффициентов полиномов. Коэффициенты матрицы ${ }^{T} W V$ могут быть выписаны

$$
{ }^{T} W V=\left(\begin{array}{ccc}
-f_{1} & -f_{1} & -f_{1} \\
f_{2} & f_{2} & f_{1}^{2}-2 f_{2} \\
\left(-f_{1} f_{2}+3 f_{3}-\sqrt{D}\right) / 2 & \left(-f_{1} f_{2}+3 f_{3}+\sqrt{D}\right) / 2 & -f_{1}^{3}+3 f_{1} f_{2}-3 f_{3}
\end{array}\right) .
$$

(Представление для $\left({ }^{T} W V\right)_{3,1}$ и $\left({ }^{T} W V\right)_{3,2}$ получается, если заметить, что их сумма симметричный многочлен от корней $f_{i}$, а разность

$$
\left({ }^{T} W V\right)_{3,2}-\left({ }^{T} W V\right)_{3,1}=\left(\lambda_{3,1}-\lambda_{3,2}\right)\left(\lambda_{3,2}-\lambda_{3,3}\right)\left(\lambda_{3,3}-\lambda_{3,1}\right)
$$

с учетом порядка нумерации корней равна корню из дискриминанта $D_{i}$.) Так как матрица $S_{0}^{-1}$ рациональна, поле $K_{i}$ нормально в точности тогда, когда дискриминант $D_{i}$ 
уравнения (3) - квадрат рационального числа. Учитьвая, что $D_{5}=13^{2}, D_{6}=273^{2}$, $D_{7}=7^{2}$, получаем полиномы, даюшие представление корней $\phi_{i, j}\left(\lambda_{i, k}\right)=\lambda_{i, j+k}$ :

$$
\begin{aligned}
\phi_{5,1}(\lambda) & =2-2 \lambda-\lambda^{2}, & \phi_{5,2}(\lambda) & =-3+\lambda+\lambda^{2}, & & \phi_{5,3}(\lambda)=\lambda, \\
3 \phi_{6,1}(\lambda) & =10-6 \lambda-\lambda^{2}, & 3 \phi_{6,2}(\lambda) & =-22+3 \lambda+\lambda^{2}, & & \phi_{6,3}(\lambda)=\lambda, \\
\phi_{7,1}(\lambda) & =2+\lambda-\lambda^{2}, & \phi_{7,2}(\lambda) & =-2 \lambda+\lambda^{2}, & & \phi_{7,3}(\lambda)=\lambda .
\end{aligned}
$$

По тройкам алгебраических чисел (5) определим пары разложимых кубических форм $g_{i}(X), g_{i}^{*}(X), X \in \mathbb{R}^{3}$, которые назовем сопряжсенными друг другу:

$$
\begin{gathered}
g_{i}(X)=L_{i, 1} X L_{i, 2} X L_{i, 3} X=\prod_{j=1}^{3} e_{j} L_{i} X \\
g_{i}^{*}(X)={ }^{T} \widetilde{L}_{i, 1} X^{T} \widetilde{L}_{i, 2} X^{T} \widetilde{L}_{i, 3} X=\prod_{j=1}^{3}{ }^{T} X L_{i}^{-1} e_{j}=\prod_{j=1}^{3} e_{j}{ }^{T} L_{i}^{-1} X .
\end{gathered}
$$

Коэффициенты форм - рациональные числа (они не меняются при перестановках корней $\lambda_{i, j}$ ). Введем нормированные формы $\widetilde{g}_{i}=c_{i} g_{i}, \widetilde{g}_{i}^{*}=c_{i}^{*} g_{i}^{*}, c_{i}, c_{i}^{*} \in \mathbb{Z}$, принимающие в целых точках $X \in Z^{3}$ взаимно простые в совокупности целые значения. Однородные кубические формы от $X={ }^{T}\left(x_{1}, x_{2}, x_{3}\right) \in \mathbb{R}^{3}$ можно записать как линейные формы относительно 10-мерных векторов $Y$ с компонентами-мономами:

$$
\begin{aligned}
& Y \stackrel{\text { def }}{=} T\left(x_{1}^{3}, x_{1}^{2} x_{2}, x_{1}^{2} x_{3}, x_{1} x_{2}^{2}, x_{1} x_{2} x_{3}, x_{1} x_{3}^{2}, x_{2}^{3}, x_{2}^{2} x_{3}, x_{2} x_{3}^{2}, x_{3}^{3}\right), \\
& \widetilde{g}_{5}(X)=1 g_{5}(X)=(1,-1,1,-4,-5,-4,-1,3,10,1) Y, \\
& \widetilde{g}_{5}^{*}(X)=169 g_{5}^{*}(X)=(5,-4,3,-5,1,-2,-1,-1,4,-1) Y, \\
& \widetilde{g}_{6}(X)=7 g_{6}(X)=(7,35,-7,28,7,-28,-9,-31,-12,19) Y, \\
& \widetilde{g}_{6}^{*}(X)=1183 g_{6}^{*}(X)=(-7,42,-35,7,49,-28,-9,-23,4,9) Y, \\
& \widetilde{g}_{7}(X)=1 g_{7}(X)=(1,6,2,5,8,-1,1,1,-9,-1) Y, \\
& \widetilde{g}_{7}^{*}(X)=392 g_{7}^{*}(X)=(-8,24,-8,-10,16,2,1,-1,-9,1) Y .
\end{aligned}
$$

Спектром формы назовем значения, принимаемые формой в целых точках $\mathbb{Z}^{3} \backslash\{0\}$. После умножения на некоторое рациональное число спектр разложимой формы будет лежать в $\mathbb{Z}$, поэтому он дискретен. Эквивалентными будем считать формы

$$
h_{k}(X)=\prod_{j=1}^{3} L_{k, j} X, \quad L_{k, j} \in \mathbb{R}_{*}^{3} \backslash\{0\}, \quad k=1,2
$$

если определяюшие формы векторы $L_{k, j}$ переводятся друг в друга заменой переменных $Y=C X$ с унимодулярной матрицей $C$ (целочисленной с определителем \pm 1 ). То есть $L_{1, j} C=d_{s(j)} L_{2, s(j)}, j=1,2,3$, где $d_{j} \neq 0$, a $s$ - перестановка индексов $\{1,2,3\}$, принадлежащая группе Галуа уравнения (3). Так как $h_{2}(X)=h_{1}(C X) / \prod_{j=1}^{3} d_{j}$, спектры 
эквивалентных форм $h_{1}(C X)$ и $h_{2}(X)$ пропорциональны, а определяемые формами многогранники Клейна переводятся отображением $C$ друг в друга:

$$
C: K_{\sigma_{1}, \sigma_{2}, \sigma_{3}}^{(1)} \rightarrow K_{\operatorname{sign}\left(d_{1}\right) \sigma_{t(1)}, \operatorname{sign}\left(d_{2}\right) \sigma_{t(2)}, \operatorname{sign}\left(d_{3}\right) \sigma_{t(3)}}^{(2)}
$$

(здесь $t=s^{-1}$ - перестановка, обратная перестановке $s$ ). Тем же свойством обладают относительные минимумы: целочисленные точки $X$ октанта, удовлетворяющие (2), перейдут в целые точки соответствующего октанта, удовлетворяющие аналогичным условиям.

Чтобы преобразование осуществляло автоморфизм совокупности многогранников Клейна разложимой формы, необходимо (не достаточно) вьполнение ряда свойств.

1. Коэффициенты $(M)_{j, k}$ матрищы преобразования суть рациональные числа: целые точки - вершины многогранников - переводятся $M$ в целые точки.

2. Преобразование сохраняет объемы, $\operatorname{det} M= \pm 1$ : объемы как опирающейся на грань пирамиды П с вершиной в нуле, так и ее образов $M^{k} \Pi, k \in \mathbb{Z}$, с точностью до множителя $1 / 3$ ! целые.

3. Наконец, октанты переходят в октанты, т.е. три задающие октанты плоскости $\left\{X: e_{j} L X=0\right\}$ переходят друг в друга. Следовательно, $M$ в композиции $M U$ с некоторой перестановкой $U$ октантов переводит задающие октанты векторы $e_{j} L$ в пропорциональные. Векторы $e_{j} L$ - собственные векторы $A$, поэтому матрица $M U$ выражается через $A$ (как квадратичньй полином $p(A))$.

Свойства 2,3 означают в точности, что формы (10) инвариантны относительно $M$.

Покажем, что матрице $U$ могут отвечать не любые перестановки, а только элементы групш Галуа алгебраического поля $K_{i}$. Пусть, например, матрища $U$ оставляет на месте один из векторов $\left(e_{1} L\right)$ и меняет местами два других $\left(e_{2} L, e_{3} L\right) ; J^{(23)}$ - матрица перестановки векторов $e_{2}$ и $e_{3}$. Предположим при этом, что матрища $M$ имеет рациональные коэффициенты. Так как

$$
\begin{aligned}
M & =p(A) U=L^{-1} p(\Lambda) J^{(23)} L=B^{-1} W^{-1} p(\Lambda) W W^{-1} J^{(23)} W B \\
& =B^{-1} p\left(A_{0}\right) W^{-1} J^{(23)} W B,
\end{aligned}
$$

то $B M B^{-1}=p\left(A_{0}\right) W^{-1} J^{(23)} W$. Для присоединенной матрицы $A_{0}$ верно $A_{0}^{j-1}{ }^{T} e_{k}=$ $A_{0}^{k-1} e_{j}, j, k=1,2,3$, поэтому справедливо

$$
\left(\sum_{j=1}^{3} p_{j} A_{0}^{j-1}\right) Q=\left(\sum_{j=1}^{3} q_{j} A_{0}^{j-1}\right) P, \quad P={ }^{T}\left(p_{1}, p_{2}, p_{3}\right), \quad Q={ }^{T}\left(q_{1}, q_{2}, q_{3}\right) \in \mathbb{R}^{3}
$$

Возьмем в (12) за $P$ набор коэффициентов многочлена $p$, а за $Q$ - первьй столбец матрицы $W^{-1} J^{(23)} W: Q={ }^{T}\left(s_{0}, s_{1}, s_{2}\right)$. Тогда слева получим первьй столбец $F$ рациональной матрицы $B M B^{-1}$, а в правой части - ненулевую целочисленную матрицу $G=$ $\sum_{j=0}^{2} s_{j} A_{0}^{j}$. Определитель матрищы $G$ не равен 0 (иначе бы многочлен $\sum_{j=0}^{2} s_{j} \lambda^{j}$ делил неприводимьй характеристический многочлен $f(\lambda)$ матрищы $A_{0}$ ), а сама матрица обратима. Поэтому рациональны коэффициенты вектора $P=G^{-1} F$ (числа $p_{j}$ ) и, следовательно, коэффициенты матрищы $p\left(A_{0}\right)$. Итак, рациональность $M$ эквивалентна 
рациональности $p\left(A_{0}\right)^{-1} B M B^{-1}=W^{-1} J^{(23)} W$. Получено противоречие, поскольку, например, коэффициент

$$
\left(W^{-1} J^{(23)} W\right)_{2,2}=\lambda_{i, 1}^{2}+2 \lambda_{i, 2} \lambda_{i, 3}=-2 f_{i, 2}-2 f_{i, 1} \lambda_{i, 1}-\lambda_{i, 1}^{2}
$$

иррационален.

Покажем, что случай, когда $U$ - циклическая перестановка базисных векторов, возможен. Положим $U_{i} \stackrel{\text { def }}{=} L_{i}^{-1} J L_{i}$. Рациональность элементов матрицы

$$
U_{i}=B_{i}^{-1} S_{i, 0}^{-1 T} W_{i} J W_{i} B_{i}
$$

эквивалентна рациональности

$$
{ }^{T} W_{i} J W_{i}=\left(\begin{array}{ccc}
3 & -f_{i, 1} & f_{i, 1}^{2}-2 f_{i, 2} \\
-f_{i, 1} & f_{i, 2} & \left(-f_{i, 1} f_{i, 2}+3 f_{i, 3}+\sqrt{D_{i}}\right) / 2 \\
f_{i, 1}^{2}-2 f_{i, 2} & \left(-f_{i, 1} f_{i, 2}+3 f_{i, 3}-\sqrt{D_{i}}\right) / 2 & -2 f_{i, 1} f_{i, 3}+f_{i, 2}^{2}
\end{array}\right)
$$

и снова означает, что поле $\mathbb{Q}\left(\lambda_{i, j}\right)$ нормально. Тождество $U_{i} \phi_{i, j}\left(A_{i}\right)=\phi_{i, j+1}\left(A_{i}\right) U_{i}$, эквивалентное соотношению $J \phi_{i, j}\left(\Lambda_{i}\right) J^{-1}=\phi_{i, j+1}\left(\Lambda_{i}\right)$, показьвает, что матрица $U_{i}$ не коммутирует с полиномами от $A_{i}$ и поэтому не выражается через $A_{i}$.

Из $(3),(4)$ следует, что $\operatorname{det}\left(\phi_{i, j}\left(A_{i}\right)\right)=f_{i, 3}= \pm 1$, и отображения $\phi_{i, j}\left(A_{i}\right), j=1,2,3$, - автоморфизмы разложимых форм. Вьпишем матрицы автоморфизмов $A_{i}, \phi_{i, 1}\left(A_{i}\right), U_{i}$ :

$$
\begin{aligned}
& A_{5}=\left(\begin{array}{ccc}
0 & 2 & 1 \\
1 & -2 & 0 \\
0 & 1 & 1
\end{array}\right), \quad \phi_{5,1}\left(A_{5}\right)=\left(\begin{array}{ccc}
0 & -1 & -3 \\
0 & 0 & -1 \\
-1 & -1 & -1
\end{array}\right), \quad U_{5}=\left(\begin{array}{ccc}
1 & 0 & 1 \\
0 & 0 & 1 \\
0 & -1 & -1
\end{array}\right), \\
& A_{6}=\left(\begin{array}{ccc}
1 & 2 & 1 \\
-1 & -5 & 4 \\
2 & 5 & 0
\end{array}\right), \quad \phi_{6,1}\left(A_{6}\right)=\left(\begin{array}{ccc}
1 & -3 & -5 \\
-2 & -1 & -1 \\
-3 & -3 & -4
\end{array}\right), \quad U_{6}=\left(\begin{array}{ccc}
1 & 2 & 1 \\
0 & 0 & -1 \\
0 & 1 & -1
\end{array}\right), \\
& A_{7}=\frac{1}{2}\left(\begin{array}{ccc}
0 & -2 & -2 \\
1 & 5 & 1 \\
-1 & -1 & -1
\end{array}\right), \quad \phi_{7,1}\left(A_{7}\right)=\frac{1}{2}\left(\begin{array}{ccc}
4 & 2 & -2 \\
-1 & -2 & 0 \\
-1 & 0 & 2
\end{array}\right), \quad U_{7}=\frac{1}{2}\left(\begin{array}{ccc}
2 & 8 & 0 \\
0 & -1 & 1 \\
0 & -3 & -1
\end{array}\right) \text {. }
\end{aligned}
$$

4. Сопряженные многогранники пятой и шестой форм. Пусть форма $g$ эквивалентна форме

$$
\widehat{g}(X)=\prod_{j=1}^{3} e_{j} \widehat{L} X
$$

а строки $e_{j} \widehat{L}$ матрицы $\widehat{L}$ являются собственными векторами симметричной матрицы $\widehat{A}$, $\widehat{A}=\widehat{L}^{-1} \Lambda \widehat{L}$. Последнее условие равносильно тому, что матрица $M=\widehat{L}^{T} \widehat{L}$ диагональна. Тогда форма $\widehat{g}(X)$ эквивалентна своей сопряженной форме

$$
\widehat{g}(X)=\prod_{j=1}^{3} e_{j} \widehat{L}^{T} \widehat{L}^{T} \widehat{L}^{-1} X=\prod_{j=1}^{3} e_{j}(M)_{j, j}{ }^{T} \widehat{L}^{-1} X=\operatorname{det} M \widehat{g}^{*}(X)
$$


Тем же свойством обладает и форма $g$. Так как $\widehat{g}(C X)=g(X)$, то $\widehat{g}^{*}\left({ }^{T} C^{-1} X\right)=g^{*}(X)$ и $g(X)=\widehat{g}(C X)=\operatorname{det} M \widehat{g}^{*}(C X)=\operatorname{det} M^{\sim} g^{*}\left({ }^{T} C C X\right)$.

Для формы $g_{5}$ возьмем $\widehat{L}_{5}=W \widehat{B}_{5} \mathrm{c}$

$$
\widehat{B}_{5}=\left(\begin{array}{ccc}
1 & -1 & 0 \\
0 & 2 & 1 \\
0 & 1 & 0
\end{array}\right)
$$

тогда получим

$$
\widehat{A}_{5}=\left(\begin{array}{ccc}
0 & 0 & 1 \\
0 & 1 & 1 \\
1 & 1 & -2
\end{array}\right), \quad C_{5}=\widehat{B}_{5}^{-1} B_{5}, \quad{ }^{T} C_{5} C_{5}=\left(\begin{array}{ccc}
1 & 0 & -1 \\
0 & 1 & 0 \\
-1 & 0 & 2
\end{array}\right)
$$

и $\operatorname{det} M=\operatorname{det}^{2} \widehat{L}_{5}=\left(\operatorname{det} W_{5} \operatorname{det} \widehat{B}_{5}\right)^{2}=D_{5}=13^{2}$. Итак, формы $g_{5}$ и $g_{5}^{*}$ эквивалентны:

$$
g_{5}(X)=169 g_{5}^{*}\left(x_{1}-x_{3}, x_{2},-x_{1}+2 x_{3}\right) .
$$

Для шестой экстремальной формы целочисленной симметричной матрицы с характеристическим уравнением $f_{6}(\lambda)=0$ не существует, и приходится действовать иначе, чем для пятой формы.

Для эквивалентности форм $h_{1}=g(X)$ и $h_{2}=g^{*}(X)$ достаточно, чтобы матрица $M=L C^{T} L$ была диагональной или переставленной диагональной матрицей $M=$ $\operatorname{diag}\left\{m_{j}\right\}_{j=1}^{3} J^{l}, l \in\{0,1,2\}$, т.е.

$$
M=\sum_{k=0}^{2} p_{k} \Lambda^{k} U^{l}, \quad p_{k} \in \mathbb{R}
$$

Следовательно,

$$
C={ }^{T} L M L={ }^{T} B{ }^{T} W M W B={ }^{T} B S_{0}\left(\sum_{k=0}^{2} p_{k} A_{0}^{k}\right) U^{l} B .
$$

Задача свелась к нахождению трех чисел $p_{k}$ таких, что определитель матрицы

$$
{ }^{T} B \sum_{k=0}^{2} p_{k} S_{k} U^{l} B
$$

равен \pm 1 , а все ее элементы - целые числа. Первое свойство дает кубическое относительно $p_{k}$ уравнение

$$
\begin{aligned}
\pm 1= & D \operatorname{det} B^{2} \prod_{j=1}^{3} \sum_{k=0}^{2} p_{k} \lambda_{j}^{k} \\
= & D \operatorname{det} B^{2}\left\{p_{0}^{3}-f_{1} p_{0}^{2} p_{1}+\left(f_{1}^{2}-2 f_{2}\right) p_{0}^{2} p_{2}+f_{2} p_{0} p_{1}^{2}\right. \\
& \left.+\left(3 f_{3}-f_{1} f_{2}\right) p_{0} p_{1} p_{2}-f_{3} p_{1}^{3}+\left(f_{2}^{2}-f_{1} f_{3}\right) p_{0} p_{2}^{2}+f_{1} f_{3} p_{1}^{2} p_{2}-f_{2} f_{3} p_{1} p_{2}^{2}+f_{3}^{2} p_{3}^{3}\right\}
\end{aligned}
$$


$\left(D=\operatorname{det} S_{0}\right.$ - дискриминант уравнения $\left.f(\lambda)=0\right)$. Из второго условия следует, что элементы матрицы $\sum_{k=0}^{2} p_{k} A_{0}^{k}$ - рациональные числа со знаменателями, делящими произведение квадрата знаменателя $b$ определителя матрищы $B^{-1}$ на $(3-l)$-ю степень знаменателя определителя матрицы $U$ и на $D$ - общий знаменатель элементов $S_{0}^{-1}$.

Возьмем для формы $g_{6}$ матрицу

$$
C=C_{6}=\left(\begin{array}{ccc}
6 & -1 & -4 \\
-1 & 2 & 0 \\
-4 & 0 & 3
\end{array}\right)
$$

получим $M={ }^{T} L_{6}^{-1} C_{6} L_{6}^{-1}=\operatorname{diag}\left\{\left(76+12 \lambda_{6, j}-3 \lambda_{6, j}^{2}\right) / 13\right\}_{j=1}^{3}, \operatorname{det} M=169$. Отсюда находим $g_{6}(C X)=169 g^{*}(X)$ или

$$
\widetilde{g}_{6}\left(6 x_{1}-x_{2}-4 x_{3},-x_{1}+2 x_{2},-4 x_{1}+3 x_{3}\right)=\widetilde{g}_{6}^{*}\left(x_{1}, x_{2}, x_{3}\right) \text {. }
$$

Таким образом, доказана

Теорема 1. Для пятой и шестой әкстремальных форм многогранники Клейна әквивалентны сопряженным многогранникам Клейна.

5. Группы отображений и фундаментальные области. Изучим теперь структуру поверхностей $\partial K_{i}$. Для пятой и шестой форм рассмотрим грушшы $G_{i}^{+}, i=5,6$, преобразований вида

$$
A_{i}^{2 j} \phi_{i, 1}\left(A_{i}\right)^{2 k} U_{i}^{l} X_{0}, \quad j, k \in \mathbb{Z}, \quad l=0,1,2 .
$$

Мы видели, что многогранники $K_{i,+++}$ относительно них инвариантны. Укажем фундаментальные области $H_{i}$ относительно действия данных групп. Вычислим методом [12] поверхности $\partial K_{i,+++}$. Для формы $g_{5}$ с каждой вершиной $X, \widetilde{g}_{5}(X)=1$, многогранника Клейна сопряжены шесть точек: по три точки со значением формы $\widetilde{g}_{5}$, равными 1 и 5 . Для формы $g_{6}$ точкам $X$ поверхности многогранника Клейна, значения формы $\widetilde{g}_{6}(X)$ в которых равно 7, сопряжены точки со значениями формы в них 9 и 33 . Если $\widetilde{g}_{6}(X)=9$, то в сопряженной $X$ точке $Y$ значения $\widetilde{g}_{6}(Y)$ равны 9,19 или 33 . Если $\widetilde{g}_{6}(X)=19$, то в сопряженной точке $\widetilde{g}_{6}(Y)=9$. Наконец, если $\widetilde{g}_{6}(X)=33$, то в сопряженных точках форма $\widetilde{g}_{6}(Y)$ равна 7 или 9 . Введем для некоторых найденньх точек обозначения

$$
\begin{gathered}
P_{5,1}=(1,0,0), \quad P_{5,2}=(2,-2,1), \quad P_{5,3}=(3,1,1), \quad P_{5,4}=(4,1,-2), \\
P_{6,1}=(1,0,0), \quad P_{6,2}=(1,12,-3), \quad P_{6,3}=(22,3,15), \quad P_{6,4}=(43,-15,-12) .
\end{gathered}
$$

Отображениями $A_{i}, \phi_{i, 1}\left(A_{i}\right), U_{i}$ они переводятся друг в друга:

$$
\begin{gathered}
P_{i, 2}=A_{i}^{2} P_{i, 1}, \quad P_{i, 3}=\phi_{i, 1}\left(A_{i}\right)^{2} P_{i, 1} \\
P_{i, 4}=\phi_{i, 2}\left(A_{i}\right)^{2} P_{i, 1}, \quad P_{i, 4}=U_{i} P_{i, 3}=U_{i}^{2} P_{i, 2} .
\end{gathered}
$$


Обозначим $\Gamma_{5, j}^{(2)}$ треугольные части граней поверхностей $\partial K_{5,+++}$ :

$$
\begin{array}{ll}
\Gamma_{5,1}^{(2)}=\{(1,0,0),(2,-2,1),(3,-3,1) / 3\}, & \rho=1, \\
\Gamma_{5,2}^{(2)}=\{(1,0,0),(2,-2,1),(7,-1,4) / 3\}, & \rho=1,
\end{array}
$$

и через $\Gamma_{6, j}^{(2)}$ обозначим трех- и четырехугольные грани (части граней) поверхности $\partial K_{6,+++}$ :

$$
\begin{array}{ll}
\Gamma_{6,1}^{(2)}=\{(1,0,0),(4,-1,0),(13,-5,-3),(8,-3,-2)\}, & \rho=1, \\
\Gamma_{6,2}^{(2)}=\{(8,-3,-2),(78,-31,-19),(122,-48,-30) / 3\}, & \rho=2, \\
\Gamma_{6,3}^{(2)}=\{(13,-5,-3),(8,-3,-2),(78,-31,-19),(58,-23,-14)\}, & \rho=3, \\
\Gamma_{6,4}^{(2)}=\{(4,-1,0),(13,-5,-3),(58,-23,-14)\}, & \rho=2, \\
\Gamma_{6,5}^{(2)}=\{(4,-1,0),(58,-23,-14),(88,-33,-18) / 3\}, & \rho=1, \\
\Gamma_{6,6}^{(2)}=\{(78,-31,-19),(505,-201,-123),(58,-23,-14)\}, & \rho=3 .
\end{array}
$$

(В скобках приведены расстояния граней, частями которых являются области.)

Поверхность многогранника Клейна кубической разложимой формы удобно рассматривать в логарифмических координатах $l_{j}=l_{j}(X)=\ln \left|L_{j} X\right|, j=1,2,3$. Там она представляется как двумерная периодическая поверхность, параллельная плоскости $l_{1}+l_{2}+l_{3}=0$. Еегруппа автоморфизмов преврашается в группу движений, сохраняющую указанную плоскость. В естественных координатах $l_{3}^{\prime}=l_{1}+l_{2}+l_{3}, l_{1}^{\prime}=l_{1}-l_{3}^{\prime} / 3$, $l_{2}^{\prime}=l_{2}-l_{3}^{\prime} / 3$, некоторые свойства симметрии поверхности многогранника Клейна теряют наглядность, поэтому удобнее взять координаты $n_{i}$, связанные с $l_{i}$ ортогональньми преобразованиями. Для расположенных вне плоскостей $\mathscr{L}_{j}, j=1,2,3$, точек $X$ положим

$$
n_{1}=\frac{l_{1}+l_{2}+l_{3}}{\sqrt{3}}, \quad n_{2}=\frac{-l_{1}+2 l_{2}-l_{3}}{\sqrt{6}}, \quad n_{3}=\frac{l_{1}-l_{3}}{\sqrt{2}} .
$$

На рис. 1-4 в координатах $n_{2}, n_{3}$ показаны поверхности многогранников Клейна $\partial K_{i,+++}$ и относительные минимумы для форм $g_{5}, g_{6}, g_{7}$ и $g_{7}^{*}$ соответственно. Целочисленные точки поверхностей (вершины и точки на ребрах и гранях) на рисунках закрашены, а точки относительных минимумов - нет. Над точками надписаны значения, принимаемые в них целочисленными формами $\widetilde{g}_{i}, \widetilde{g}_{i}^{*}$, а рядом с некоторыми точками - их координаты. Границы областей $\Gamma_{i, j}^{(2)}$, из которых составлены фундаментальные области, проведены жирньми линиями. Отображения $U_{5}, U_{6}$ вьглядят как повороты плоскости $n_{2}, n_{3}$ вокруг начала координат на угол $2 \pi / 3$ по часовой стрелке, а отображения $A_{i}^{2}$ и $\phi_{i, 1}\left(A_{i}\right)^{2}$ действуют как сдвиги на векторы $\overline{P_{i, 1} P_{i, 2}}$ и $\overline{P_{i, 1} P_{i, 3}}$ соответственно. Многогранные области $H_{5}=\Gamma_{5,1}^{(2)} \cup \Gamma_{6,2}^{(2)}, H_{6}=\bigcup_{j=1}^{6} \Gamma_{6, j}^{(2)}$ будут фундаментальными областями относительно групш (14). Осталось показать, что данные группы исчерпьвают все автоморфизмы соответствующих поверхностей многогранников Клейна $\partial K_{i,+++}$. 


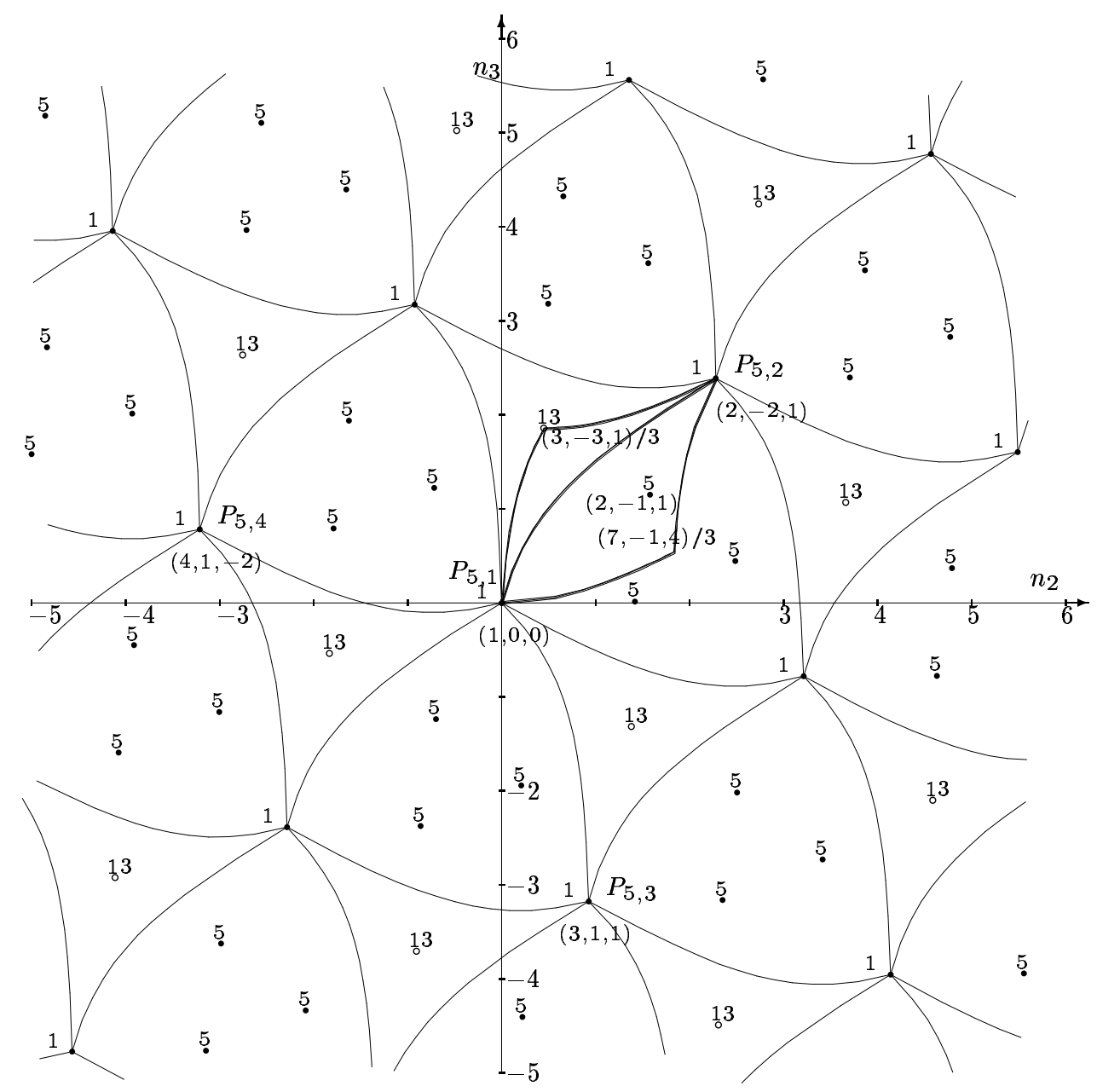

Рис. 1. Многогранник Клейна формы $g_{5}$

Для любого автоморфизма $h$ формы $g_{5}$ композиция $h^{\prime}$ автоморфизма $h$ с некоторьм преобразованием вида (14) оставляет на месте грань $\Gamma_{5,2}^{(2)}$. Учитывая, какие значения принимает форма $g_{5}$ в целых точках опирающегося на $\Gamma_{5,2}^{(2)}$ конуса, получаем, что $h^{\prime}$ переводит ее вершины $P_{5,1}$ и $P_{5,2}$ друг в друга, а вершину треугольника $P_{5,5}=(7,-1,4) / 3$ и целочисленную точку $P_{5,6}=(2,-1,1)$ этой грани оставляет на месте. Поскольку площади треугольных частей граней с вершинами в точках $P_{5,1}, P_{5,5}, P_{5,6}$ и $P_{5,2}, P_{5,5}, P_{5,6}$ различны (их отношение равно 3 ), единственное такое отображение - тождественное. Для многогранника шестой формы аналогичньй вьвод можно сделать, взяв точки

$$
P_{6,5}=(13,-5,-4), \quad P_{6,6}=(24,-9,-5), \quad P_{6,7}=(51,-20,-12) .
$$

Точка $P_{6,5}$ - это вершина треугольной грани $\Gamma_{6,4}^{(2)}$ расстояния 2 многогранника, причем значение $\widetilde{g}_{6}\left(P_{6,5}\right)=19$ не равно значениям формы $g_{6}$ в других вершинах грани. Две 


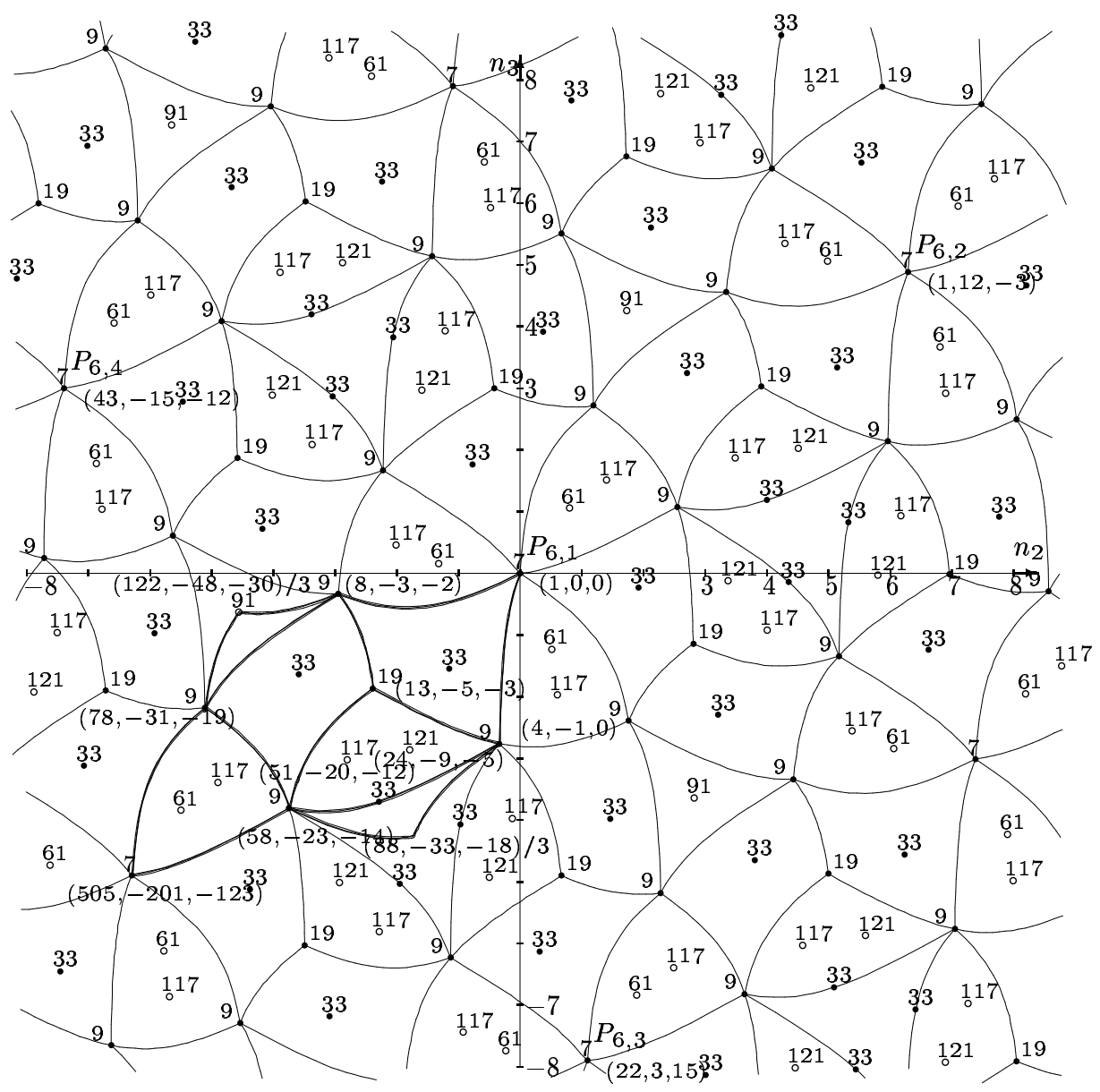

Рис. 2. Многогранник Клейна формы $\widetilde{g}_{6}$

другие точки суть расположенные под этой гранью точки относительного минимума формы, значения формы $\widetilde{g}_{6}$ в которых различны и равны 121 и 117.

Осталось заметить, что многогранники Клейна разных полостей переводятся друг в друга преобразованиями $A_{i}, \phi_{i, 1},-E$. Таким образом, установлено следующее свойство многогранников Клейна пятой и шестой экстремальных форм.

ТЕорема 2. Для форм $g_{5}, g_{6}$ группы автоморфизмов их многогранников Клейна имеют две образующ,х бесконечного порядка $A_{i} u \phi_{i, 1}\left(A_{i}\right)$ и две образующих $U_{i}$ и-Е порядков 3 и 2 соответственно:

$$
A_{i}^{j} \phi_{i, 1}\left(A_{i}\right)^{k} U_{i}^{l} X_{0}, \quad j, k \in \mathbb{Z}, \quad l=0,1,2, \quad i=5,6 .
$$

Фундаментальная область $H_{5}$ совокупности поверхностей многогранников Клейна $\bigcup_{\Sigma} \partial K_{5, \Sigma}$ состоит из треугольников $\Gamma_{5,1}^{(2)}, \Gamma_{5,2}^{(2)}$ расстояния 1 , а фундаментальная область $H_{6}$ поверхностей многогранников Клейна $\bigcup_{\Sigma} \partial K_{6, \Sigma}$ состоит из тре- 


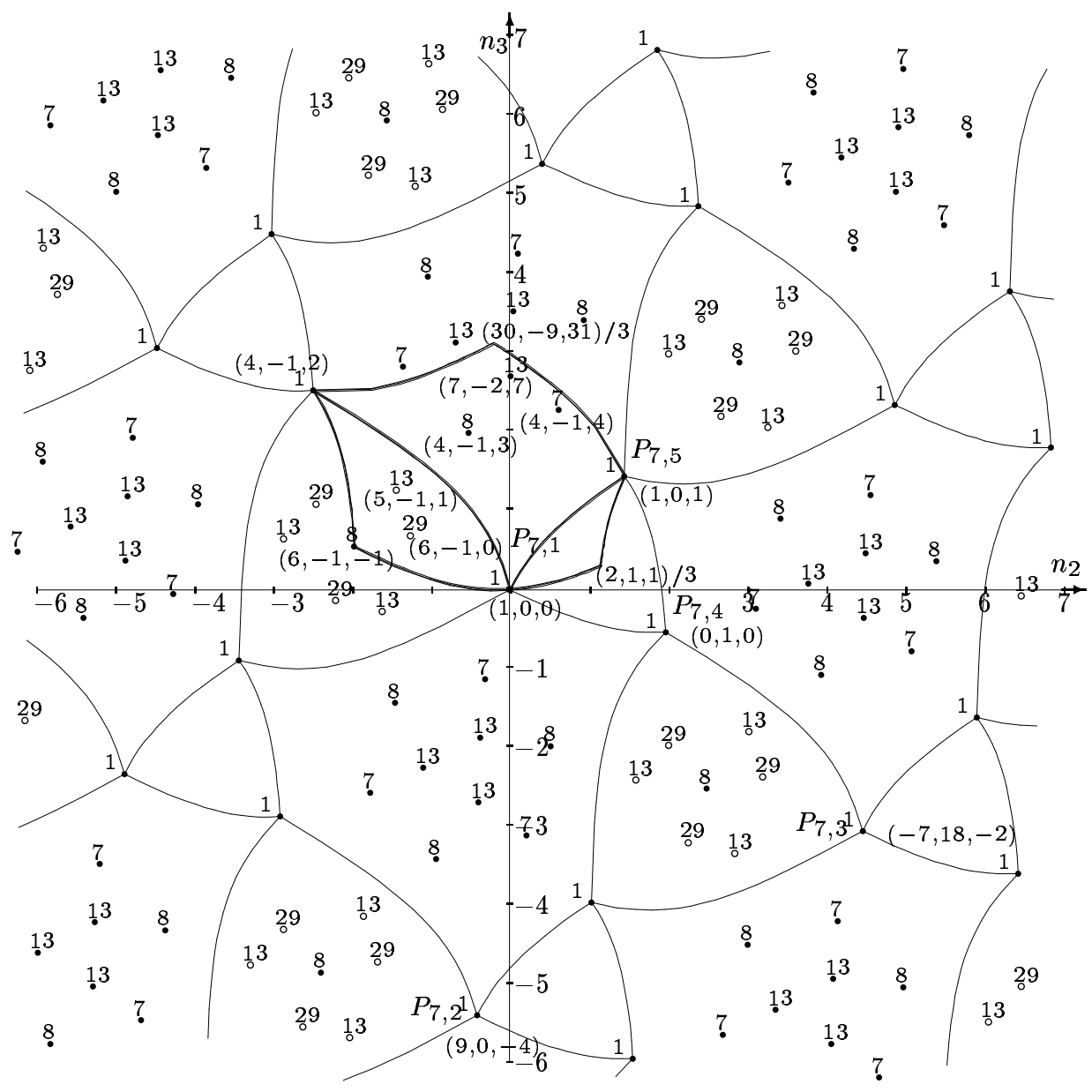

Рис. 3. Многогранник Клейна формы $\widetilde{g}_{7}$

угольника $\Gamma_{6,4}^{(2)}$ и двух четырехугольников $\Gamma_{6,1}^{(2)}, \Gamma_{6,3}^{(2)}$ расстояния 1 , двух треугольников $\Gamma_{6,2}^{(2)}, \Gamma_{6,5}^{(2)}$ расстояния 2 и треугольника $\Gamma_{6,6}^{(2)}$ расстояния 3.

Кольцо с единицей

$$
O=\left\{\sum_{k=0}^{2} p_{k} A_{0}^{k}, p_{k} \in \mathbb{Z}\right\}
$$

одновременно является $\mathbb{Z}$-модулем, т.е. $O$ это порядок поля $G L(3, \mathbb{Q})$. Для матрицы $B \in G L(3, \mathbb{Q})$, если матрица $A=B^{-1} A_{0} B$ не унимодулярна, сопряженный с $O$ порядок

$$
O_{B}=B^{-1} O B=\left\{\sum_{k=0}^{2} p_{k} A^{k}, p_{k} \in \mathbb{Z}\right\}
$$

состоит не только из унимодулярных матриц. Содержашиеся в порядке $O_{B}$ множества матриц с определителем \pm 1 (множество $S O_{B}$ ) и множества унимодулярных матриц $\left(O_{B, 1}\right)$ - коммутативные группы по умножению. Групша $S O_{B}$, а следовательно, 


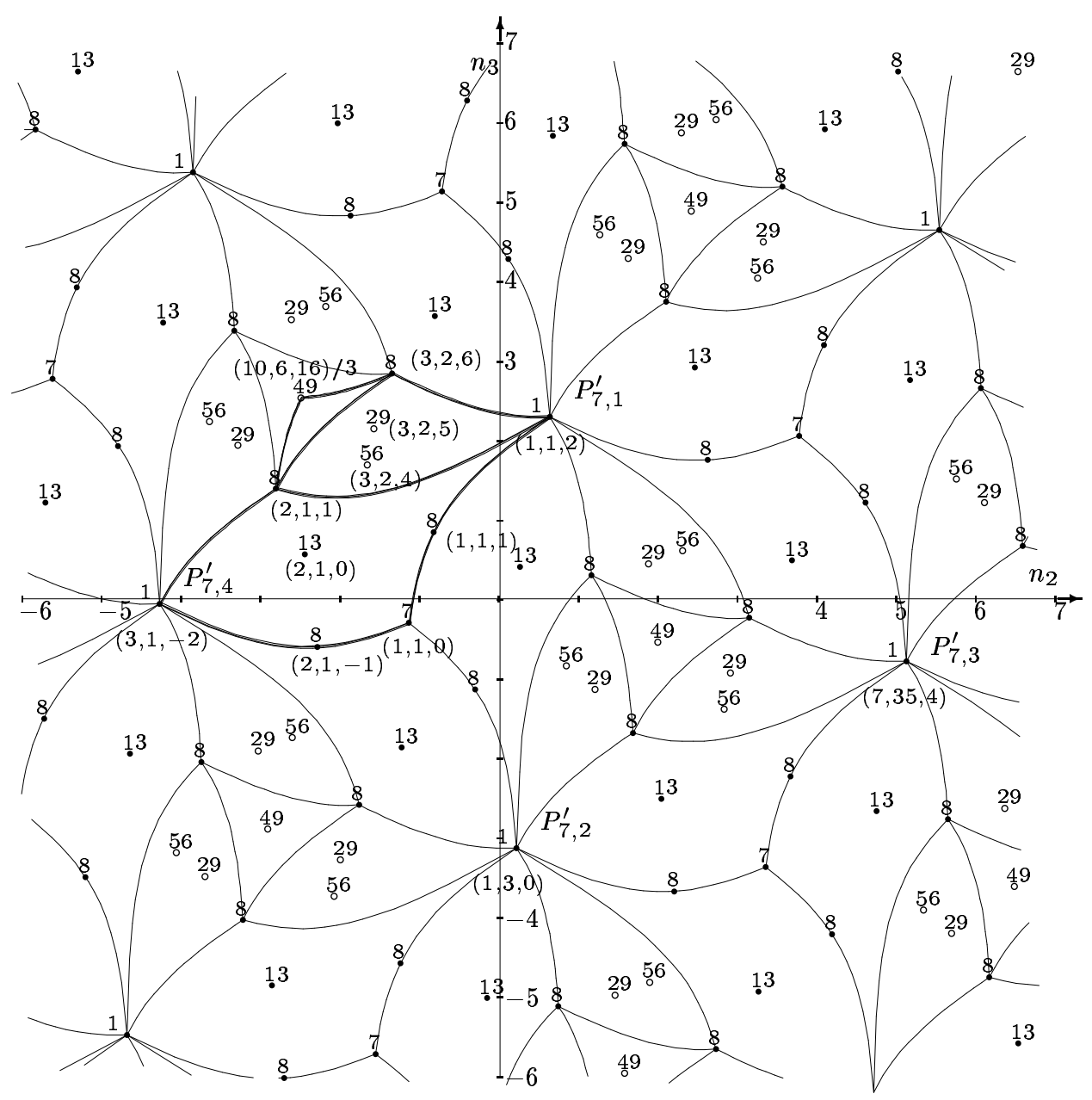

Рис. 4. Многогранник Клейна формы $\widetilde{g}_{7}^{*}$

и $O_{B, 1}$, как ее подгруппа конечного индекса,- свободные группы с двумя образуюшими (см. [21, гл. II, §2]).

Для седьмой экстремальной формы можно проверить, что индекс подгрупшы $O_{B_{7}, 1}$ в группе $O_{B_{7}}$ равен 7 . Точнее, матрицы $A_{7}^{j} \phi_{7,1}\left(A_{7}\right)^{k}$ унимодулярны, если показатели степеней $j, k$ удовлетворяют условию $2 j+k \equiv 0(\bmod 7)$. Отсюда следует, что матрицы $A_{7}^{j} \phi_{7,1}\left(A_{7}\right)^{k} U_{7}^{l}$ унимодулярны, если для $j, k \in \mathbb{Z}, l \in\{0,1,2\}$ выполнено $2 j+k(3 l+1)-$ $2 l \equiv 0(\bmod 7)$. Образующими в последней группе будут матрицы

$$
F_{1}=A_{7}^{2} \phi_{7,1}\left(A_{7}\right)^{3}=3 E+2 A_{7}-2 A_{7}^{2}, \quad F_{2}=A_{7}^{3} \phi_{7,1}\left(A_{7}\right)=E-2 A_{7}^{2}, \quad R=A_{7}^{2} U_{7}^{2} .
$$

Для формы $g_{7}$ введем обозначения некоторых областей $\Gamma_{7, j}^{(2)}-$ граней или частей граней многогранников $\partial K_{7,++}, \partial K_{7,+++}^{*}$ : 


$$
\begin{array}{rlrl}
\Gamma_{7,1}^{(2)} & =\{(1,0,0),(2,1,1) / 3,(1,0,1)\}, & \rho=1, \\
\Gamma_{7,2}^{(2)} & =\{(1,0,0),(1,0,1),(30,-9,31) / 3,(4,-1,2)\}, & \rho=1, \\
\Gamma_{7,3}^{(2)} & =\{(6,-1,-1),(1,0,0),(4,-1,2)\}, & \rho=3, \\
\Gamma_{7,1}^{(2) *} & =\{(1,1,0),(1,1,2),(2,1,1),(3,1,-2)\}, & \rho=1, \\
\Gamma_{7,2}^{(2) *}=\{(2,1,1),(3,2,6),(10,6,16) / 3\}, & \rho=2, \\
\Gamma_{7,3}^{(2) *}=\{(2,1,1),(1,1,2),(3,2,6)\}, & \rho=1,
\end{array}
$$

являющихся выпуклыми оболочками приведенных точек. Здесь полные грани суть $\Gamma_{7,1}^{(2) *}, \Gamma_{7,3}^{(2) *}$, а области $\Gamma_{7,2}^{(2)}, \Gamma_{7,2}^{(2) *}$ имеют вершинами не целые точки. Определим также множества $H_{7}=\bigcup_{j=1}^{3} \Gamma_{7, j}^{(2)}, H_{7}^{*}=\bigcup_{j=1}^{3} \Gamma_{7, j}^{(2) *}$.

Все точки поверхности $\partial K_{7,+++}$ могут быть получены из точек множества $H_{7}$ при помощи автоморфизмов многогранника, составляющих групу

$$
G_{7}^{+} \stackrel{\text { def }}{=}\left\{F_{1}^{2 i} F_{2}^{2 j} R^{l}, j, k \in \mathbb{Z}, l=0,1,2\right\} \text {. }
$$

Чтобы показать, что группа автоморфизмов исчерпьвается группой $G_{7}^{+}$, установим, что множество $H_{7}$ будет фундаментальной областью поверхности многогранника. Для этого достаточно показать, что во множестве $H_{7}$ нет подобластей, переводящихся друг в друга не вошедшими в грушу $G_{7}^{+}$автоморфизмами многогранника Клейна. Но сформулированное свойство следует из того, что всякий автоморфизм множества $H_{7}$ должен переводить в себя единственную грань $\Gamma_{7,1}$ расстояния 3 , а расположенные за этой гранью точки относительных минимумов - в аналогичные точки за этой же гранью. В единственных содержащихся там точках относительных минимумов $(5,-1,1),(6,-1,0)$ форма $\widetilde{g}_{7}$ принимает значения 29 и 13 , а в вершине $(6,-1,-1)$ - значение 8 . Поскольку все три перечисленных значения формы $\widetilde{g}_{7}$ различны, рассматриваемый автоморфизм $H_{7}-$ это тождественное преобразование.

Группа автоморфизмов совокупности поверхностей всех восьми многогранников Клейна $\bigcup_{\Sigma} \partial K_{7, \Sigma}$ получается из $G_{7}^{+}$добавлением преобразований $F_{1}$ и $-E$. Сопряженньй многогранник Клейна имеет сопряженную группу автоморфизмов, а фундаментальной областью его поверхности будет множество $H_{7}^{*}$. Итак, доказана

ТЕОрема 3. Для формы $g_{7}$ (соответственно $g_{7}^{*}$ ) группа автоморфизмов $G_{7}$ (соответственно $\left.G_{7}^{*}\right)$ имеет две образующих бесконечного порядка $F_{1}, F_{2}\left({ }^{T} F_{1},{ }^{T} F_{2}\right)$ и две образующих $R u-E\left({ }^{T} R u-E\right)$ порядков 3 и 2 соответственно. Фундаментальная область $H_{7}$ совокупности поверхностей многогранников Клейна $\bigcup_{\Sigma} \partial K_{7, \Sigma}$ состоит из двух треугольников $\Gamma_{7,1}^{(2)}, \Gamma_{7,3}^{(2)}$ расстояний 1 и 3 и четырехугольника $\Gamma_{7,2}^{(2)}$ расстояния 1 , а фундаментальная область $H_{7}^{*}$ совокупности поверхностей многогранников Клейна $\bigcup_{\Sigma} \partial K_{7, \Sigma}^{*}$ состоит из двух треугольников $\Gamma_{7,2}^{(2) *}, \Gamma_{7,3}^{(2) *}$ расстояний 2 и 3 и четырехугольника $\Gamma_{7,1}^{(2)}$ расстояния 1.

На рис. 3,4 изображены поверхности многогранники Клейна форм $g_{7}, g_{7}^{*}$. Как и раньше, выделены фундаментальные области. Также для некоторой точки $P_{7,1}=(1,0,0) \in$ 
$\partial K_{7,+++} P_{7,1}^{\prime} \in \partial K_{7,+++}^{*}$ на рис. 3 отмечены ее образы $P_{7, j}$, впервые попадающие на тот же многогранник $\partial K_{7,+++}$ под действием степеней базисных отображений

$$
P_{7,2}=F_{1}^{2} P_{7,1}, \quad P_{7,3}=F_{2}^{2} P_{7,1}, \quad P_{7,4}=R P_{7,1}, \quad P_{7,5}=R^{2} P_{7,1} .
$$

Аналогично на рис. 4 отмечены

$$
P_{7,1}^{\prime}=(1,1,2), \quad P_{7,2}^{\prime}={ }^{T} F_{1}^{2} P_{7,1}^{\prime}={ }^{T} U P_{7,1}^{\prime}, \quad P_{3}^{\prime}={ }^{T} F_{2}^{2} P_{7,1}^{\prime}, \quad P_{4}={ }^{T} U^{2} P_{7,1}^{\prime} .
$$

В таблице 1 представлены значения форм $\widetilde{g}_{7}, \widetilde{g}_{7}^{*}$ во взаимно сопряженных точках.

\begin{tabular}{|c|c|c|c|c|}
\hline Форма & $\begin{array}{c}\text { Значение } \\
\text { формы } \\
\text { в точке }\end{array}$ & $\begin{array}{c}\text { Расположение } \\
\text { точки }\end{array}$ & $\begin{array}{c}\text { Сопря- } \\
\text { женная } \\
\text { форма }\end{array}$ & $\begin{array}{c}\text { Значения } \\
\text { сопряженной формы } \\
\text { в сопряженных точках }\end{array}$ \\
\hline$\widetilde{g}_{7}$ & $\begin{array}{c}1 \\
7 \\
8 \\
13\end{array}$ & $\begin{array}{l}\text { вершина } \\
\text { на грани } \\
\text { на грани } \\
\text { на грани }\end{array}$ & $\widetilde{g}_{7}^{*}$ & $\begin{array}{c}1,1,7,8,8,8,13 \\
1 \\
1 \\
1\end{array}$ \\
\hline$\widetilde{g}_{7}^{*}$ & $\begin{array}{c}1 \\
7 \\
8 \\
8 \\
13\end{array}$ & $\begin{array}{l}\text { вершина } \\
\text { вершина } \\
\text { вершина } \\
\text { на ребре } \\
\text { на грани }\end{array}$ & $\widetilde{g}_{7}$ & $\begin{array}{c}1,1,1,1,1,1,7,7,7 \\
8,8,8,13,13,13 \\
1,1,1 \\
1 \\
1,1 \\
1\end{array}$ \\
\hline
\end{tabular}

ТАБЛИЦА 1

6. Разложения по матричным алгоритмам. Исследовался вопрос, насколько улавливают структуру поверхностей многогранников Клейна различные многомерные матричные обобщения разложения в цепную дробь. Вычислялись разложения кратного вектора формы по алгоритмам Эйлера, Якоби (четыре варианта), Пуанкаре, Бруна, Брюно, Парусникова (см. [11], [13]). При этом в [11], [13] алгоритм Брюно описан неверно. Правильное описание его шага такое: для тройки чисел $\alpha \geqslant \beta \geqslant \gamma \geqslant 0$ вычисляются $a=[\alpha / \beta], b=[\beta / \gamma], c=[(\alpha-a \beta) / \gamma]([\cdot]$ - целая часть), делаются преобразования $\alpha^{\prime}=\alpha-a \beta-c \gamma, \beta^{\prime}=\beta-b \gamma, \gamma^{\prime}=\gamma$, после чего числа $\alpha^{\prime}, \beta^{\prime}, \gamma^{\prime}$ упорядочиваются. Также использовался ускоренньй алгоритм Бруна, отличающийся тем, что последовательные шаги, где нет переупорядочения компонент векторов, включаются в шаг с упорядочением: шаг - это получение и упорядочение чисел $\alpha^{\prime}=\alpha-[\alpha / \beta] \beta, \beta^{\prime}=\beta$, $\gamma^{\prime}=\gamma$.

Рассматривался начальньй вектор $V$ (вектор-столбец) с положительньпи компонентами. Для его подходящих дробей (определение см. ниже) $X$ вычислялись значения форм $g_{i}(X)$. Дополнительным индексом $k$ пометим остаток $V_{k}$ цепной дроби - вектор, возникающий на $k$-м шаге матричного алгоритма; положим $V_{-1}=V$. Остатки связаны соотношениями $V_{k}=Q_{k} L_{k-1}$, где унимодулярные матрищы $Q_{k}$ определяются конкретным алгоритмом. Периодичность алгоритма - это пропорциональность двух разных по номеру остатков, а длина периода - это разность первых таких номеров шагов. 
Введем пять матриц перестановок. Матрицу $\widetilde{Q}_{0}=\widetilde{Q}_{0}(V)$ возьмем такой, чтобы компоненты $\widetilde{Q}_{0} V$ убьвали, и положим

$Q^{(123)}=E, \quad Q^{(132)}=\left(\begin{array}{ccc}1 & 0 & 0 \\ 0 & 0 & 1 \\ 0 & 1 & 0\end{array}\right), \quad Q^{(213)}=\left(\begin{array}{lll}0 & 1 & 0 \\ 1 & 0 & 0 \\ 0 & 0 & 1\end{array}\right), \quad Q^{(312)}=\left(\begin{array}{lll}0 & 0 & 1 \\ 1 & 0 & 0 \\ 0 & 1 & 0\end{array}\right)$.

Для всех алгоритмов, кроме Якоби, положим $Q_{0}=\widetilde{Q}_{0}$, а для четырех вариантов алгоритмов Якоби положим $Q_{0}=Q^{(\kappa)} \widetilde{Q}_{0}, \kappa=123,132,213,312$. Подходящими дробями назовем векторы $N_{k}{ }^{T} e_{j}, j=1,2,3, k=0,1, \ldots$, где $N_{k}=\left(Q_{k} \ldots Q_{1} Q_{0}\right)^{-1}$.

В качестве начальных векторов взяты векторы $V={ }^{T} L_{i, 3}$ и $\widetilde{L}_{i, 3}$, отвечающие наибольшим корням $\lambda_{i, 3}$ уравнений (3). Предпочтение этим векторам было отдано потому, что для первых экстремальных форм $g_{i}(X), g_{i}^{*}(X), i \leqslant 6$, у них не было отрищательных компонент и первые шаги матричных алгоритмов не требовали модификации. Ниже приведены результаты разложений не для всех матричньх алгоритмов, а только для которых подходящие дроби не уходят глубоко под поверхности многогранников Клейна (на данном начальном векторе).

Для вектора $L_{5,3} \approx(1,1.3772,2.6511)$ ускоренный алгоритм Бруна имеет период $T_{1}=3 A_{5}^{2}+7 A_{5}-2 E=A_{5}^{2} \phi_{5,1}\left(A_{5}\right)^{2}$ длины 5 , алгоритмы Якоби (123) и (132) - период $T_{2}=8 A_{5}^{2}+19 A_{5}-6 E=-A_{5}^{2} \phi_{5,1}\left(A_{5}\right)^{3}$ длины 5 , алгоритм Брюно - период $T_{3}=2 A_{5}^{2}+5 A_{5}-E=A_{5} \phi_{5,1}\left(A_{5}\right)^{2}$ длины 2 , а алгоритм Парусникова - период $T_{4}=$ $9931 A_{5}^{2}+23608 A_{5}-7211 E=-A_{6} \phi_{5,1}\left(A_{5}\right)^{9}$ длины 8. Для вектора ${ }^{T} \widetilde{L}_{5,3} \approx(.2866$, $.0849, .2250)$ алгоритм Бруна имеет период ${ }^{T} T_{3}$ длины 4 , алгоритмы Якоби $(213),(132)$ и $(312)$ - период ${ }^{T} T_{2}$ длины 5 , алгоритм Брюно - период $106^{T} A_{5}^{2}+252^{T} A_{5}-77 E=$ $-{ }^{T} A_{5}^{4} \phi_{5,1}\left({ }^{T} A_{5}\right)^{5}$ длины 7 и алгоритм Парусникова - период ${ }^{T} T_{4}$ длины 7.

Для вектора $L_{6,3} \approx(1,1.3583,1.8853)$ алгоритм Бруна и алгоритмы Якоби $(123)$, $(213)$ и (132) имеют период $T_{5}=17 A_{6}^{2}+126 A_{6}+5 E=A_{6}^{2} \phi_{6,1}\left(A_{6}\right)^{2}$. Длины периодов у этих алгоритмов разные: для алгоритма Бруна - 12 , а для алгоритмов Якоби - 8 шагов. Алгоритм Брюно имеет наиболее короткий период (в 3 шага) $T_{6}=\left(2 A_{6}^{2}+15 A_{6}+E\right) / 3=$ $-A_{6} \phi_{6,1}\left(A_{6}\right)$. В разложении вектора ${ }^{T} \widetilde{L}_{6,3} \approx(.2338, .1232, .3176)$ период ${ }^{T} T_{5}$ был найден у алгоритмов Бруна (длины 12), Якоби (213), (132) и (312) (длины 8), Брюно (длины 4).

В разложении вектора $L_{7,3} \approx(1,5.0489, .5550)$ у алгоритма Бруна был найден период $T_{7}=2 A_{7}^{2}-E=-A_{7}^{2} \phi_{7,1}\left(A_{7}\right)$ длины 2 . У алгоритма Брюно период не был обнаружен, но все найденные по алгоритму подходящие дроби были точками многогранников Клейна или относительными минимумами. Вместо вектора $\widetilde{L}_{7,3} \approx(-.0863, .2178$, -.0239) брался вектор $V=L_{7,3}^{*}$ с положительными компонентами - абсолютньми значениями компонент $\widetilde{L}_{7,3}$. Поскольку у первой и третьей компонент сменили знаки, для исследования периодичности использовалась не матрица $A_{7}$, а связанная с ней матрища $A_{7}^{*}={ }^{T}\left(Z^{-1} A_{7} Z\right)$, где $Z=\operatorname{diag}\{-1,1,-1\}$ - диагональная матрица замен знаков. Периодичньми оказались только алгоритмы Якоби (132) и Брюно. У алгоритма Брюно периодом длины 1 служит матрища $T_{7}^{*}={ }^{T}\left(Z^{-1} T_{7} Z\right)=2\left(A_{7}^{*}\right)^{2}-E=-\left(A_{7}^{*}\right)^{3} \phi_{7,1}\left(A_{7}^{*}\right)$, а у алгоритма Якоби (132) - ее квадрат (период длины 7) $T_{8}=16\left(A_{7}^{*}\right)^{2}+4 A_{7}^{*}-7 E=$ $\left(A_{7}^{*}\right)^{6} \phi_{7,1}\left(A_{7}^{*}\right)^{2}$.

Автор благодарит А. Д. Брюно за постановку задачи и полезные замечания. 


\section{СПИСОК ЦИТИРОВАННОЙ ЛИТЕРАТУРЫ}

[1] Хинчин А.Я. Цепные дроби. М.: Физматгиз, 1961.

[2] Klein F. Ueber eine geometrische Auffassung der gewöhnlichen Kettenbruchentwicklung // Nachr. Ges. Wiss. Göttingen Math. -Phys. Kl. 1895. № 3. P. 357-359.

[3] Klein F. Sur une representation géometrique du developpement en fraction continue ordinaire // Nouv. Ann. Math. (3). 1896. V. 15. P. 321-331.

[4] Klein F. Ausgewählte Kapitel der Zahlentheorie. Bd. I. Einleitung. Vorlesung 1895/1896 herausgegeben von A. Sommerfeld. Göttingen, 1896.

[5] Koksma J. F. Diophantische Approximationen. Berlin: Springer, 1936.

[6] Davenport H. On the product of three homogeneous linear forms. I // Proc. London Math. Soc. (2). 1938. V. 44. P. 412-431.

[7] Davenport H. Note on the product of three homogeneous linear forms // J. London Math. Soc. 1941. V. 16. P. 98-101.

[8] Davenport H. On the product of three homogeneous linear forms. IV // Proc. Cambridge Philos. Soc. 1943. V. 39. P. 1-21.

[9] Swinnerton-Dyer H.P.F. On the product of three homogeneous linear forms // Acta Arith. 1971. V. 18. P. 371-385.

[10] Брюно А. Д., Парусников В.И. Многогранники Клейна для двух кубических форм Давенпорта // Матем. заметки. 1994. Т. 56. № 4. С. 9-27.

[11] Брюно А. Д., Парусников В.И. Сравнение разньх обобщений цепных дробей // Матем. заметки. 1997. Т. 61. № 3. С. 339-348.

[12] Parusnikov V.I. Klein polyhedra for complete decomposable forms // Number Theory / ed. K. Győry, A. Pethő and V. T. Sós. Berlin-New York: de Gruyter, 1998. P. 453-463.

[13] Парусников В.И. Многогранники Клейна для четвертой экстремальной кубической формы // Матем. заметки. 2000. Т. 67. №1. С. 110-128.

[14] Парусников В.И.Многогранники Клейна для пятой экстремальной кубической формы. Препринт ИПМ им. М.В. Келдыша РАН №69. М., 1998.

[15] Парусников В. И. Многогранники Клейна для шестой экстремальной кубической формы. Препринт ИПМ им. М.В. Келдыша РАН №69. М., 1999.

[16] Парусников В.И. Многогранники Клейна для седьмой экстремальной кубической формы. Препринт ИПМ им. М.В. Келдыша РАН № 79. М., 1999.

[17] Charve L. De la reduction des formes ternaires positives et de leur application aux irrationelles du troisieme degre // Ann. Sci. Ecole Norm. Sup. Ser. 2. Supplement. 1880. V. 9. P. 1-156.

[18] Коркина Е. И.Двумерные цепные дроби. Самые простые примеры // Особенности гладких отображений с дополнительньми структурами / ред. В.И. Арнольд. Тр. МИАН. Т. 209. М.: Физматлит, 1995. С. 143-166.

[19] Голдман А., Таккер А. Многогранные выпуклые конусы // Линейные неравенства. М.: ИЛ, 1959. С. 142-161.

[20] Брюно А. Д. Локальньй метод нелинейного анализа дифференциальных уравнений. М.: Наука, 1979.

[21] Боревич З. И., Шафаревич И. Р. Теория чисел. М.: Наука, 1972.

Институт прикладной математики им. М. В. Келдьша РАН

Поступило

E-mail: parus@spp.keldysh.ru

15.01 .2002

Исправленный вариант

26.11 .2004 